\title{
Die Gletschertöpfe im Gletschergarten von Luzern
}

\begin{abstract}
Dieses Kapitel vermittelt einen kleinen Ausschnitt aus älterer und neuerer volkstümlicher und wissenschaftlicher Literatur, die sich mit der Erklärung der Phänomene im Gletschergarten beschäftigt hat. Die heute aktuelle Lehrmeinung wird nur in knapper Form zusammenfassend dargestellt, da diese bereits in anderen Publikationen ausführlich dargestellt worden ist (vgl. Ebers 1952, Klebelsberg 1948, Roesli 1957).
\end{abstract}

\section{Warum weite Kreise an einer heute überholten Theorie festhalten}

Die erste gedruckte Erklärung zu den aufgedeckten Gletschertöpfen Luzern erschien am 23. November 1872 im „Luzerner Tagblatt” . . Es sind dies nach der Ansicht der Naturwissenschafter aus der Gletscherzeit stammende, hie und da vorkommende sogenannte Strudellöcher, welche beim Abschmelzen eines Gletschers durch den herabstürzenden Wasserstrahl nach und nach ausgehöhlt wurden ..." Diese Interpretation entspricht durchaus der heutigen Ansicht über die Entstehung der Gletschertöpfe. Leider ist nicht bekannt, wer diese Auskunft damals dem Tagblatt gab. Es dürfte sich aber wahrscheinlich nur um den Geologen Dr. F. J. Kaufmann oder um den Apotheker Dr. Stierlin aus Luzern gehandelt haben. Dass das Phänomen „Gletschergarten" sofort richtig gedeutet wurde, überrascht uns heute nicht. In der Schweiz steckte man zu dieser Zeit in der ersten grossen Gletscher- und Eiszeitforschungsperiode, da man teilweise den Gletschern noch alles zumutete, ob es sich nun um das Abtragen ganzer Berge oder das Aushobeln tiefster Becken handelte. Die eigentliche Eiszeittheorie war noch nicht alt. Erst 1834 brachte der Vortrag von J. Charpentier über erratische Blöcke und Gletschertöpfe an der Tagung der Schweizerischen Naturforschenden Gesellschaft in Luzern den Durchbruch des Eiszeitgedankens in der Schweiz. In Deutschland wurde diese Erkenntnis sogar erst 40 Jahre später offiziell anerkannt. Schon vor der Entdeckung der Gletschertöpfe in Luzern waren solche aus Skandinavien und in der Schweiz bei Bex und Sitten bekannt.

Drei Wochen nach der eingangs zitierten Pressemeldung berichtete die "Zürcher Freitagszeitung” über den Besuch des Dozenten Albert Heim bei den Luzerner Riesentöpfen. Es wurde erklärt, dass der
Eiswasserfall Steine in Drehbewegung versetzt und dieselben den Sandstein ausgehöhlt hätten. Albert Heim benutzte in allen seinen späteren Arbeiten $(1874,1883,1885,1919)$ den Begriff „Mahlstein” für die in den Gletschertöpfen aufgefundenen abgerundeten Findlinge. Die von Heim unterstützte Theorie mit den „Mahlsteinen” wurde nun im Gletschergarten im wahrsten Sinne des Wortes ,in Stein geprägt". Man achtete bei den folgenden Ausgrabungen streng darauf, dass pro Gletschertopf je einer der vielen Findlinge, die ursprünglich darin lagen, im Topf belassen wurde. In den grossen Strudellöchern liegt seither ein grosser, in den kleinen ein kleiner erratischer Block. Durch die Errichtung des Modells „künstliche Gletschermühle” im Jahre 1896 wurde diese Hypothese des „mahlenden Blockes” noch veranschaulicht und damit noch tiefer in das Bewusstsein der Besucher geprägt. So beständig wie sich der hohle „Mahlstein” in der künstlichen Gletschermühle unter dem Wasserstrahl dreht, so hartnäckig erhielt sich auch diese Theorie bis heute in Laien-, aber auch in Fachkreisen. Obwohl W. Amrein schon 1938 und F. Roesli 1957 in ihren Publikationen recht deutlich aufzeigten, dass diese Erklärung nicht mit den im Gletschergarten entstandenen Erosionsformen in Einklang gebracht werden kann, erscheint der ,rotierende Findling" sogar noch in den neuesten einheimischen Schulbüchern. Neben den oben erwähnten Autoren aus dem Gletschergarten haben aber auch andere Wissenschaftler wie R. v. Klebelsberg (1948) und E. Ebers (1952) betont, dass allein die Schleifwirkung des feinen Gesteinsmaterials im Schmelzwasser oder sogar nur das reine Wasser für die Entstehung der Gletschertöpfe verantwortlich ist. Andererseits hat R. Staub noch 1952 in der Beschreibung der "Gletschermühlen" von Maloja die Wichtigkeit der „Mahlsteine” hervorgehoben.

Leider wird der unglücklich gewählte Begriff „Gletschermühle" immer wieder falsch verwendet. In der Literatur hat sich schon lange eingebürgert, dass eine „Gletschermühle” ein Eisschacht oder ein -system ist, in welches das Schmelzwasser von der Gletscheroberfläche hinunterstürzt. Das Resultat dieser Gletschermühle, die mit dem Zurückschmelzen des Gletschers verschwindet, ist die in den Untergrund geschliffene Hohlform unter dem Gletschereis und wird "Gletschertopf" (glacial pot hole, marmite) genannt. 
Wie zu erwarten war, sind im Verlauf der letzten hundert Jahre unterschiedlichste, zum Teil recht merkwürdige „Theorien” über die Bildung dieser „Riesentöpfe” entstanden. So wurden „Amreins Löcher" im Gletschergarten urweltlichen Riesen oder auch geschäftstüchtigen Betrügern zugeschrieben. Auf das oft Verwirrung stiftende Nebeneinandervon Zeugen der Miozänzeit und der Eiszeit im Gletschergarten ist der Bericht des „Vaterland” vom 26. November 1872 zurückzuführen: „... Vielleicht mögen sie (die Aushöhlungen) sich durch Erosion von darüberflutendem Wasser gebildet haben zur Zeit, da durch die Hebung der Alpen und der subalpinen Molasse das vorher bis an die Alpen reichende Molassemeer zurückgedrängt wurde, oder dann können sie auch eine Strandbildung dieses Molassemeeres und durch die Brandung desselben entstanden sein.” Schon „eiszeitlicher” waren die Vermutungen, dass sich das Gletschereis selber in den Fels hineingebohrt habe. Der Luzerner Leo Erni (1945) stellte sich einen See auf der Eisoberfläche vor, der ähnlich einem Badewannenabfluss durch einen Eisschacht nach unten entleert worden sei. Die dabei entstandenen Wirbel hätten sich dann in den unten anstehenden Fels eingeprägt.

\section{Entstehung der Gletschertöpfe}

Die in Flussbetten und am Fuss von Wasserfällen auftretenden Kolklöcher erinnern in vielem an die Gletschertöpfe. Die Entstehungsbedingungen sind ja auch sehr ähnlich, und es ist manchmal sogar für den geübten Beobachter recht schwierig, eiszeitliche Gletschertöpfe von gewöhnlichen, meist jüngeren Flusskolken zu unterscheiden.

Die steil stehenden Spülrinnen an den Wandungen der Gletschertöpfe zeigen, dass die Kolke das Werk eines steil herabstürzenden Wasserstrahls sind. Die $\mathrm{zu}$ beobachtenden eleganten Erosionsformen des grössten Gletschertopfes im Gletschergarten - mit den sich oft überschneidenden Rinnen, mit den anscheinend zufällig verteilten Aushöhlungen und Erhebungen an den Wänden und einer scharf aufragenden Rippe im Zentrum - schliesst die Wirkung eines schweren rotierenden Findlings zum vornherein aus.

Die Wucht des mit Sand, Kies und gröberem Geröll vermischten niederstürzenden Schmelzwassers besass genügend Energie, um Fels jeder Härte auszuhöhlen. Nur während der eiszeitlichen Vergletscherung standen die genügenden Wassermengen und die notwendigen Höhendifferenzen zur Verfügung, um die Bildung solcher Riesentöpfe zu ermöglichen. Eine Entstehung im Präglazial oder im frühen Postglazial kann man aus diesen und verschiedenen andern Gründen, die hier nicht näher erläutert werden, ausschliessen.
Das Schmelzwasser, das sich ungehindert auf der eiszeitlichen Gletscheroberfläche zwischen dem Alpenrand und der Gegend von Luzern zu reissenden Bächen sammeln konnte, verschwand plötzlich in einer Gletscherspalte. Im Innern des Gletschers stürzten die Wassermassen durch einzelne senkrechte Schächte oder ganze Schachtsysteme hinunter. Dabei wurden die Schächte durch Thermoerosion noch erweitert. Dem wahrscheinlich schon trüben Schmelzwasser wurde noch zusätzlich Gesteinsmaterial zugeführt, das vorher im Gletschereis eingefroren war. In einem mehr oder weniger steilen Einfallswinkel prallte dieses Wasser auf das Bett des Gletschers, den Sandsteinfelsen von Luzern. Das Vorrücken des Gletschers und damit der Eisschächte hatte zur Folge, dass sich auch der Einfallswinkel des Wasserstrahls ständig änderte. Durch weiter bergwärts liegende Aufbrüche von neuen Gletscherspalten wurden die tätigen Gletschermühlen ihres Wassers beraubt und damit stillgelegt. Aber nicht nur durch neue Spalten wurde der Auskolkungsvorgang unterbrochen. Schmelzwasser stand nur im Sommer in genügenden Mengen zur Verfügung. Während der übrigen kälteren Jahreszeiten kam nicht nur die Aushöhlung der bestehenden Gletschertöpfe zum Stillstand, sondern die Felsoberfläche wurde weiter abgeschliffen und somit die Tiefe der Kolke vermindert, bis sich zufällig wieder eine Spalte genau über dem bereits ausgestrudelten Topf öffnete. Das komplizierte Formenspiel an den Wänden des grossen Gletschertopfes spiegelt den sich ständig ändernden Einfallswinkel des Wasserstrahles wider und beweist auch die Mehrphasigkeit der Formentwicklung.

Als Beweis der Tätigkeit von Mahlsteinen wurde oft angeführt, dass die aufgefundenen schweren Findlinge meist am Grunde der Gletschertöpfe lagen. (Im grossen Gletschertopf lagen mehrere bis 6 Tonnen schwere Exemplare!) Dies ist aber leicht verständlich, denn stürzte einmal ein Block aus der Grund- oder Obermoräne in einen Topf, konnte er kaum wieder herausgespült werden. Das feinere Material konnte aber immer wieder herausgewirbelt werden. Die schweren Blöcke konnten mehrere Phasen der Topfbildung überdauert haben, während das feine Füllmaterial erst nach der letzten Stillegung der Gletschermühle liegen blieb. Leider ist nicht genau überliefert, wie das Füllmaterial, das aus den Gletschertöpfen in Luzern entfernt wurde, ausgesehen hat. Die bis heute genaueste Beschreibung des Ausgrabungsmaterials aus Gletschertöpfen lieferten Brøgger \& Reusch (1874 S. 798) von den „Riesenkesseln” bei Christiania (Norwegen): „Die Reibsteine zeigten nie eine geringere Grösse als 3"; sie waren selten ganz vollkommen ausgebildet, wenn sie mehr als 1' lang waren." Unter einem ausgebildeten „Reibstein” verstanden die oben aufgeführten Autoren ein gut gerundetes Geröll. Das 
Zitat bestätigt neben den vorgängig erwähnten Fakten die heute geläufige Theorie der Gletschertopfbildung. Die im Gletschergarten aus den Gletschertöpfen geborgenen Findlinge zeigen zum Teil durch ihre Formen, dass sie nicht im Topf abgerollt, sondern festsitzend umspült worden sind. Beigeschichteten Kieselkalkblöcken wurden die Schichten entsprechend ihrer Härte deutlich mehr oder weniger tief herauspräpariert, was im Gegensatz zu den in Bergbächen zu beobachtenden Rollblöcken steht. $\mathrm{Ob}$ das Wasser überhaupt genügend Kraft hatte, um diese Findlinge zu bewegen, steht hier nicht zur Diskussion. Immerhin wurden bei heutigen alpinen Gletschern senkrechte Schächte von gegen $200 \mathrm{~m}$ Tiefe vermessen (Agassiz erwähnte vor mehr als hundert Jahren einen solchen von $260 \mathrm{~m}$ ). Stellt man sich nun einen mittelgrossen Fluss vor, der in solche Schächte hinuntergestürzt, kann ein mächtiger Findling eventuell schon bewegt worden sein. Die Findlinge aber hatten kaum die Hohlformen der Gletschertöpfe geschaffen, ob sie nun in Bewegung waren oder festsassen.

\section{Lage der Gletscherspalten}

Die Entstehung der Gletschertöpfe ist primär an das Vorhandensein von Gletscherspalten gebunden. Gletscherspalten entstehen immer dort, wo durch Hindernisse im Untergrund oder andere durch das Relief bedingte Änderungen in der Fliessgeschwindigkeit des Gletschers Spannungen im Eis entstehen. Solche Hindernisse im Bett des Gletschers charakterisieren die meisten der bisher entdeckten Gletschertöpfe (Schlosshügel von Maloja, Talriegel von Cavaglia am Berninapass, Halde von Chur, Tiergartenhügel zwischen Sargans und Flums, Tourbillon/Sitten, Le Montet bei Bex, Grosse Schanze Bern u. a.). Auch die Molasseschichtrippen von Luzern (Sonnenberg - Bramberg - Dreilinden Hombrig) waren die Ursache, die zur Entstehung einer ganzen Anzahl von Gletschertöpfen führten. Die Lage der erwähnten Gletschertöpfe ist bezeichnenderweise ausnahmslos an Stellen gebunden, wo ein fliessendes Gewässer ohne Benützung des Gletschers kaum hingelangen konnte. Diese Lagebezeichnung ist oft das einzige Merkmal, um die Gletschertöpfe von normalen Flusskolken zu unterscheiden.

\section{Zeitliche Einstufung der Luzerner Gletschertöpfe}

Die Entstehungszeit der Luzerner Gletschertöpfe genau zu fixieren ist schwierig. Die Überlegungen des Geologen F. Roesli (1957) bilden aber sicher eine Diskussionsgrundlage zur zeitlichen Einordnung.
Eine Bildung schon während der Risseiszeit kann mit grosser Wahrscheinlichkeit ausgeschlossen werden. Ein durchschnittlicher minimaler Abschleifungsbetrag des Felsens von ca. $1 \mathrm{~cm}$ pro Jahr, wie er z. B. beim Vorstoss des Allalingletschers im Jahre 1920 auf Granit gemessen wurde (Mercanton 1926), hätte genügt, um auch die grössten Gletschertöpfe von Luzern in tiefgründig verwittertem Sandstein im Verlauf der Würmeiszeit auszuebnen.

Die maximale Eisdicke bei Luzern in der Würmeiszeit betrug ca. $550 \mathrm{~m}$ (versch. Autoren). Falls - wie heute allgemein angenommen wird - unsere Gletscher zur Eiszeit, ebenso wie heute, nicht das unterkühlte Eis der arktischen Gletscher besassen, reagierte das Eis an der Basis unter dem Einfluss der Druckwärme plastisch. Es ist also wenig wahrscheinlich, dass im Würmmaximum die Gletscherspalten bis in Tiefen von mehreren hundert Metern hinunter aufklafften. Es ist demnach anzunehmen, dass die bis auf das Sandsteinbett hinunter wirksamen Gletschermühlen erst beim Rückschmelzen des Eises wirksam wurden.

Andererseits wurde die Sandsteinoberfläche nach der Bildung der Töpfe noch stark abgeschliffen. Waren ursprünglich vielleicht einmal Abflussrinnen des aus den Töpfen überfliessenden Wassers vorhanden, sind solche nicht mehr zu erkennen. Die Schrammen im Fels verlaufen bis an die Kanten der Kolke, sind also nie vom zu- oder wegfliessenden Wasser anerodiert worden. Die geschliffene Sandsteinoberfläche wie auch die Spülrinnen an den Topfwänden zeigen, dass sich die Gletschertöpfe heute nicht in ihrer ursprünglichen Tiefe präsentieren.

Damit aber die niederstürzenden Wassermassen genügend Energie besassen, um den anstehenden Fels auszuhöhlen, muss noch eine minimale Eisdikke angenommen werden.

Sind obige Überlegungen richtig, so müssen die Gletschertöpfe im Gletschergarten Luzern in den Zeitraum kurz nach dem Triengen-(Schlieren) oder Sursee-(Zürich) Stadium (Hantke 1968) entstanden sein.

\section{Neuere Untersuchungen}

In den verwendeten neueren Publikationen konnten keine grundlegend neuen Erkenntnisse über die Bildung der Gletschertöpfe erarbeitet werden. R. Streiff-Becker (1950) versuchte die Erosionsleistung durch subglaziale Wasserläufe, die nicht unter Druck stehen, zu erklären. Seine morphologische Beweisführung stimmt aber nicht mit den Gegebenheiten in der Umgebung des Gletschergartens überein. An der norwegischen Skagerrakküste untersuchte J. Gjessing (1967) einige Gletschertöpfe auf der Leeseite von Rundhöckern. Er nimmt an, 
dass hinter den Rundhöckern die subglazial fliessenden Schmelzwasserläufe in einem turbulenten Sog gegen unten abgelenkt wurden und so die Gletschertöpfe geschaffen haben. Damit versucht Gjessing zu erklären, warum die bergseitigen Topfwände oft steiler ausgebildet sind als die talseitigen. Diese Beobachtung kann auch an den Gletschertöpfen im Gletschergarten Luzern gemacht werden, nur liegen hier die Töpfe zum grössten Teil auf der Stossseite der Rundhöcker.

In modernen glaziologischen Arbeiten (Lliboutry 1968, Iken 1972 u. a.) werden die Vorgänge in den Hohlräumen heutiger Gletscher (Spalten, Schächte, Höhlen) quantitativ untersucht. Das Ziel dieser Untersuchungen ist aber nicht die Erklärung der Gletschertopfbildung, sondern u. a. die klimatisch be-

\section{Benützte Literatur}

\section{Entdeckung und Entwicklung des Gletschergartens Luzern 1872-1972}

Schifferli M. 1972: Hundert Jahre Gletschergarten Luzern. Unveröff. Manuskript, Archiv Gletschergarten Luzern

Für diese Arbeit wurden verwendet: Literatur über die Geschichte der Stadt Luzern und über die Entwicklung des Fremdenverkehrs. Presseausschnitte, Gerichtsprotokolle, Grundbucheintragungen, Briefe, Notizen, Buchhaltungsbücher, Graphiken, Sitzungsprotokolle und Jahresberichte der Stiftung Amrein-Troller etc. aus: Zentralbibliothek Luzern, Stadtarchiv Luzern, Archiv des Gletschergartens, Nachlass von Dr. h. c. W. Amrein-Küpfer und M. Blattner-Amrein. Ergänzungen durch private Aufzeichnungen von mündlichen Überlieferungen durch Frau M. Schifferli-Amrein.

\section{Die Gletschertöpfe im Gletschergarten Luzern}

Amrein, W. 1938: Die Entstehung der Gletschermühlen. Festschrift Eugen Tatarinoff, Solothurn

Aeppli, A. 1898: Der Gletschergarten in Luzern. Schweizerische Illustr. Zeitung, 1. Jahrg. H. 22, 457-458, Zürich

Brunner, H. 1954: Die Gletschermühlen von Maloja. Leben und Umwelt, Nr. 12,264-268, Aarau

Brøgger, W. C. \& Reusch, H. H. 1874: Riesenkessel bei Christiania. Ztschr. dt.geol. Ges. 26,783-815

Ebers, E. 1950: Zwei bedeutende eiszeitliche Naturdenkmale am nördlichen Alpensaum. Leben und Umwelt, 4, 73-80, Aarau

Ebers, E. 1952: Der Gletschergarten an der Deutschen Alpenstrasse. Forsch. z. dt. Landeskunde, 75 , Remagen dingten Schwankungen der Schmelzwassserzufuhr $z u$ den Veränderungen der Fliessgeschwindigkeit des Gletschers in Beziehung zu setzen. Es ist aber gut möglich, dass aus den vorgenommenen Messungen und Berechnungen auch einmal präzisere Aussagen über die Bildungsbedingungen von Gletschertöpfen resultieren. Die festgestellten Tiefen der nahezu senkrechten Kanäle im Gletscher (Gletschermühlen) und die darin gemessenen Wasserdrucke bestätigen die bisherigen Vorstellungen, die man sich aus den Beobachtungen der eiszeitlichen Gletschertöpfe über deren Entstehung machte.

Anschrift der Verfasser: Margrit Schifferli-Amrein, Im Wygart, 6204 Sempach

Peter Wick, Denkmalstrasse 4, 6006 Luzern

Gjessing, J. 1967: Potholes in connection with plastic scouring forms. Geografiska Annaler, Vol. 49 , Ser. A, 178-187, Oslo

Hantke, R. 1968: Erdgeschichtliche Gliederung des mittleren und jüngeren Eiszeitalters im zentralen Mittelland. Ur- und frühgesch. Archäologie der Schweiz, 7-26, Zürich

Haserodt, K. 1965: Riesengletschertöpfe am Nordausgang des Kalkhochalpen-Durchbruchtals der Salzach bei Golling (Salzburg), Mitt. d. Geogr. Ges. München. H. L, 161-173, München

Heim, Albert 1874: Über den Gletschergarten in Luzern, Luzern

1883: Über den „Gletschergarten” in Luzern. Vierteljahresschr. d. Naturf. Ges. Zürich, 18, Zürich

1885: Handbuch der Gletscherkunde, Stuttgart 1919: Geologie der Schweiz, Bd. 1, Leipzig

Iken, A. 1972: Velocity variations of the White Glacier. Axel Heiberg Island Research Reports. McGill University, Montreal

Klebelsberg, R. v. 1948: Handbuch der Gletscherkunde und Glazialgeologie, Bd. 1, Wien

Lliboutry, L. A. 1968: General theory of subglacial cavitation and sliding of temperate glaciers. Journal of Glaciology, Vol. 7. No. 49. 21-58

Hercanton, P. L. 1926: Les variations périodiques des glaciers des Alpes suisses. Die Alpen II 1926. 214-240. Bern

Roesli, F. 1957: Der Gletschergarten von Luzern. Luzern im Wandel der Zeiten, H. 7, Luzern

Roesli, F. \& Wick, P. 1972: Gletschergarten Luzern, Stiftung Amrein-Troller (Beschreibung), Luzern

Staub, R. \& Daeniken, A. U. 1952: Die Passlandschaft von Maloja und die Gletschermühlen, Chur

Streiff-Becker, R. 1950: Gletschermühlen, Prisma Nr. 9, 443-448, Frauenfeld. 